\title{
Supply and demand biases in linear interindustry models
}

\author{
Antonio Manresa \\ Departament de Teoria Econòmica and CREB \\ Universitat de Barcelona, Spain \\ manresa@ub.edu \\ Ferran Sancho \\ Department of Economics \\ Universitat Autònoma de Barcelona, Spain \\ ferran.sancho@uab.cat
}

\begin{abstract}
We explore the sectoral and aggregate implications of some endogeneization rules (i.e. on value-added and final demand) which have been common in the extended demand-driven Leontief model and have been recently proposed in the supply-driven Ghosh model. Extended linear models are refinements that aim at endowing the linear models with additional general equilibrium feedbacks. We detect that these rules may give rise in these models to some allegedly pathological and biased behavior. We find that in these models sectoral or aggregate output may not follow the logical and economically expected direct relationship with some underlying endogenous variables - namely, output and value-added in the supply-driven model and output and consumption in the demand-driven model. Because of their shared mathematical structure, whatever is or seems to be pathological in the Ghosh model also has a symmetric counterpart in the Leontief model. These would not be good news for the inner consistency of these linear models and raise doubts regarding the validity of their empirical applications. To avoid such possible inconsistencies, we propose new and simple endogeneization rules that have a sound economic interpretation.
\end{abstract}

Keywords: extended linear models; demand-driven model; supply-driven model.

Acknowledgments: Support from projects ECO2009-06953, ECO2012-34046, SGR2009105 (first author), and ECO2009-11857 and SGR2009-578 (second author) is gratefully acknowledged. We are also grateful to the referees for their comments. Corresponding author: Ferran Sancho at: ferran.sancho@uab.cat 


\section{Introduction}

Both the classical Leontief (1936) and Ghosh (1958) models have been subjected to criticism because of the way they handle the role of some key variables. In Ghosh, for instance, output depends on value-added and a change in a given sector's value-added will rise output everywhere with no need for additional value-added in the rest of sectors. There is more output overall but value-added is, in general, insensitive to this fact. In Leontief, in turn, output depends on final demand (say, consumption for simplicity). Any change in final consumption will raise output everywhere but this will not get reflected in any additional demand for consumption, even though overall there is more output and income around. We may conclude that consumption is insensitive to income, a fact that also runs against standard economic logic.

These observations suggest that these features of Ghosh and Leontief traditional models are not quite realistic features of any production and consumption processes. Enter therefore the extended versions of these models to overcome these unwanted properties. In the Leontief model, we can establish a relationship between output $\mathbf{x}$ and final consumption $\mathbf{d}$ (or a part of it) by way of introducing the missing connection $\mathbf{d}=\phi_{L}(\mathbf{v})$ between consumption $\mathbf{d}$ and value-added $\mathbf{v}$. In Ghosh the relationship between output and value-added (or a part of it) is made explicit linking the required value-added in each sector to put out a unit of aggregate consumption, i.e. $\mathbf{v}=\phi_{G}(\mathbf{d})$. Figure 1 below provides a simpler graphical representation of the lines of causality in the standard (in the enclosed rectangular areas) and extended models.

Figure 1: Graphical view of the Leontief and Ghosh models.

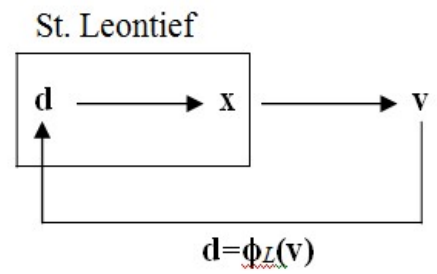

Extended Leontief

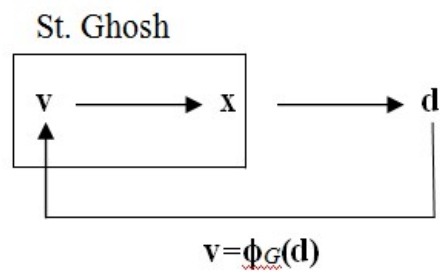

Extended Ghosh

We visually confirm why in both the standard and the extended cases, the Leontief model is demand-driven (i.e. through $\mathbf{d}$ directly, or indirectly via $\mathbf{v}$ ) and the Ghosh model is supply-driven (i.e. through $\mathbf{v}$ directly, or indirectly via $\mathbf{d}$ ).

Guerra and Sancho (2011) are, to our knowledge, the first to propose a Ghosh extended model version by incorporating value-added as an endogenous variable. They posit final consumption for each good to be a positive fraction of the corresponding sectorial output 
making the consumption coefficients fully coherent with the output allocation coefficients while sectorial value-added depends on aggregate consumption. In their version of the Ghosh model, the only exogenous variable is what we may call a public input and therefore production is driven from this supply side variable of the economy.

In a recent article, however, Oosterhaven (2012) points out an allegedly anomalous property of the so called extended Ghosh model by means of a numerical example based in the $3 \times 3$ input-output table of Guerra and Sancho (2011). The pinpointed anomaly is the possibility of having an inverse relationship between production and value-added in a sector. This means that we may have that total production in a sector increases while its value-added diminishes. This possibility is, according to Oosterhaven, even worse than leaving that variable unchanged. Furthermore, one can also see in the same example that total production of the economy may go in the opposite direction of total value-added. We share with Osterhaven that this lack of realism in the production process, may indeed be a serious criticism for any model thus built. Furthermore, this author also shows, by some numerical examples, that such an undesirable property no longer applies to the demanddriven Leontief extended model.

The main purpose of this paper is to discuss the logic and structure of these unwanted and anomalous properties - going beyond results based only on numerical examples. We do so by way of analyzing the general and explicit solutions of the equilibrium values of both the Ghosh and Leontief extended models in a $2 \times 2$ scenario, which is sufficient to identify and characterize the main issues. Since both linear models share the same mathematical structure, whatever property -good or bad- of the Ghosh model may have, we find a precise and formal counterpart in the Leontief model.

Unfortunately, what we find is that the aforementioned unwanted properties of the extended Ghosh model, i.e. the pathological behavior of a negative relationship between some sectorial production and its corresponding value-added, is unavoidable. This means that the model is biased in the sense that we will always observed this kind of behavior, at least in one sector, no matter what are the parameters of the model. Furthermore, at the aggregate level, the same anomalous relationship may be observed for some parameter values.

However, this is not the end of the story. With respect to the extended Leontief model, we can easily see from our analysis that the same troublesome relationship between production and value added, at the aggregate level, become true. Furthermore, at the sectorial level, we also show that at least for one sector we will always have a negative relationship between production and domestic demand. That systematic type of behavior makes this model biased towards a relationship (i.e. sectorial production and its domestic final demand) that economic evidence does not support.

These are bad news, because they may point to a theoretical weakening of both models, and hence of some of the results ensuing from their possible empirical applications. 
In Section 2 we present the classical Leontief and Ghosh models, in order to establish the logical introduction to Section 3, where the main results on production and consumption biases are obtained.

An open question therefore arises: is there any other way to incorporate final consumption and value-added endogenously in these models that may avoid such biased behavior? This issue is explored in Section 4 where we show that a way to avoid it in the Ghosh model is by somewhat mimicking some good features of Leontief's extended model, and the other way around. After the new rules are introduced, the aggregate problem still continuous in both models, but we establish sufficient conditions, on parameters of the models, to avoid it. Finally, Section 5 concludes.

\section{The classical L-G $2 \times 2$ models}

We will start by considering the following two accounting expressions coming from a typical (or aggregated) $2 \times 2$ input-output table:

$$
\left.\begin{array}{l}
\left.\begin{array}{l}
x(1)=z(1,1)+z(1,2)+d^{h}(1)+d^{f}(1) \\
x(2)=z(2,1)+z(2,2)+d^{h}(2)+d^{f}(2)
\end{array}\right\} \\
x(1)=z(1,1)+z(2,1)+v^{h}(1)+v^{f}(1) \\
x(2)=z(1,2)+z(2,2)+v^{h}(2)+v^{f}(2)
\end{array}\right\}
$$

All magnitudes can be considered in monetary or in (conveniently re-defined) physical units, and they are labeled in the following way:

$x(i)$ : total production in sector $i$,

$z(i, j)$ : intermediate inputs flowing from sector $i$ to sector $j$,

$d^{k}(i)$ : final demand for good $i$ with home $(k=h)$ or foreign origin $(k=f)$,

$v^{k}(i)$ : value-added applied in sector $i$ with home $(k=h)$ or foreign origin $(k=f)$.

Clearly, accounting expressions (L.1) correspond to the revenues that each sector receives according to the use or employment of its production, while accounting expressions (G.1) are the payments of each sector to the suppliers of resources used in its production process. Since revenues and payments have to be equal for each sector, we see that corresponding sums in both expressions are the same.

A matrix representation of the previous accounting balances of resources and/or payments comes in handy:

$$
\mathbf{x}=\mathbf{Z} \cdot \mathbf{e}+\mathbf{d}^{\mathrm{h}}+\mathbf{d}^{\mathrm{f}}
$$




$$
\mathbf{x}=\mathbf{Z}^{\prime} \cdot \mathbf{e}+\mathbf{v}^{\mathbf{h}}+\mathbf{v}^{\mathbf{f}}
$$

where $\mathbf{Z}$ (and its transpose, $\mathbf{Z}^{\prime}$ ) is the standard matrix of intermediate inputs flows. The column vector $\mathbf{e}$ is the unit vector and the rest of magnitudes, also column vectors, have the obvious components as appearing in the previous (L.1) and (G.1) expressions.

In order to "jump" from the previous accounting expressions to the well-known Leontief (L) and Ghosh (G) models we define the following 'technical' coefficients for output production and output allocation, respectively:

$$
\begin{aligned}
& a(i, j)=z(i, j) / x(j) \\
& b(i, j)=z(i, j) / x(i)
\end{aligned}
$$

We now introduce these coefficients into expressions (L.2) and (G.2), which will allow us to rewrite them in matrix terms as:

$$
\begin{aligned}
& \mathbf{x}=\mathbf{A} \cdot \mathbf{x}+\mathbf{d}^{\mathrm{h}}+\mathbf{d}^{\mathrm{f}} \\
& \mathbf{x}=\mathbf{B} \cdot \mathbf{x}+\mathbf{v}^{\mathrm{h}}+\mathbf{v}^{\mathrm{f}}
\end{aligned}
$$

where $\mathbf{A}=[a(i, j)]$ and $\mathbf{B}=[b(j, i)]$ are the $2 \times 2$ matrices endowed with the above 'technical' coefficients ${ }^{1}$. Notice that these coefficients are related, for all $i$ and $j$, in the following generic way:

$$
a(i, j) \cdot x(j)=z(i, j)=b(i, j) \cdot x(i)
$$

By construction, in matrix $\mathbf{A}, a(i, j)$ denotes its $i$ row and $j$ column element, its interpretation being the amount of good $i$ used to produce one unit of good $j$. In matrix $\mathbf{B}$, in contrast, $b(j, i)$ denotes its $j$ column and $i$ row element and can be interpreted as the fraction of $j$ used in the production of good $i$. The classical $\mathrm{L}$ and $\mathrm{G}$ models are those derived from (L.3) and (G.3), by assuming that coefficients in $\mathbf{A}$ and $\mathbf{B}$ are fixed parameters. The values of $\mathbf{x}$ are the unknowns of these models, which can be obtained as a function of the rest of the variables, as defined above. For exogenously given vectors $\mathbf{d}$ and $\mathbf{v}$ we can endogenously solve for production $\mathbf{x}$ and obtain the classical expressions for the Leontief and Ghosh reduced forms:

$$
\begin{aligned}
& \mathbf{x}_{L}=(\mathbf{I}-\mathbf{A})^{-1}\left(\mathbf{d}^{\mathbf{h}}+\mathbf{d}^{\mathbf{f}}\right) \\
& \mathbf{x}_{G}=(\mathbf{I}-\mathbf{B})^{-1}\left(\mathbf{v}^{\mathbf{h}}+\mathbf{v}^{\mathbf{f}}\right)
\end{aligned}
$$

\footnotetext{
${ }^{1}$ Observe that our B matrix is in fact the transposed of the standard Ghosh matrix. This allows us to have throughout the same normalized vector post-multiplication notation.
} 
Observe that the two models have the same mathematical structure and so they must share the very same properties. Furthermore, from an empirically based point of view, we have to notice that both models can use either accounting structure to solve for the same initial value $\mathbf{x}$. In other words, we may distinguish both models just because exogenous variables have different names and interpretations. As Dietzenbacher (1997) concluded: "The Ghosh models and the Leontief models can be viewed as each others mirror-image". In the next section we will see that the extended models can in fact be seen as "two faces of the same coin".

\section{The extended L-G $2 \times 2$ models}

\subsection{General considerations}

In the two classical models, the variables driving the models are exogenous, i.e. in the L model this is final demand whereas in the G model is value-added. A change in total production, due to any other change in the model characteristics, will leave such an exogenous variable unchanged. In expression (L.4) a change in foreign final demand $\mathbf{d}^{\mathbf{f}}$ will affect total output $\mathbf{x}$. Domestic demand $\mathbf{d}^{\mathbf{h}}$, however, remains surprisingly insensible to the new equilibrium output despite the economic fact that producing more output would inevitably require more primary factors, or value-added in our terminology. This extra income in the form of value-added does not have any effect on final domestic demand, again a rather surprising fact. Similarly in (G.4), a change in external value-added $\mathbf{v}^{\mathbf{f}}$ (say, through immigration in sector 1) will impact total output $\mathbf{x}$ in all sectors, but this output changes will have no effect whatsoever in the remaining levels of domestic value-added $\mathbf{v}^{\mathbf{h}}$ in both sectors. These insensibility properties of the two models put serious limitations to any model that aims to be considered either for theoretical purposes or for empirical applications and they have been widely discussed in the literature. See for instance Guerra and Sancho (2011) for the L model, and most notably Oosterhaven $(1988,1989,2012)$ and De Mesnard (2009), among others, for the G model. The joint stability issue between both models has been recently dealt with in Guerra and Sancho (2012) whereas a good elementary introduction can be found in Miller and Blair (2009, chapter 12).

A way to overcome such unwanted property has been to extend the classical models to make the exogenous variable, or more precisely some part of it, into an endogenous one. This procedure yields the so-called 'extended' L and G models in the literature. The idea is simply to add some extra conditions to the (L.4) and (G.4) equations in some sensible economic way. The traditional way to add more equations to the basic ones can be represented by the features of these two composed mappings:

$\begin{array}{lll}\text { (Extended Leontief) } & \mathbf{x} \rightarrow \mathbf{v}_{L}^{h} \rightarrow \mathbf{d}_{L}^{h} \\ \text { (Extended Ghosh) } & \mathbf{x} \rightarrow \mathbf{d}_{G}^{h} \rightarrow \mathbf{v}_{G}^{h}\end{array}$


In the Leontief case, with variables denoted with the $L$ submarker, we have that final demand is a function of output $\mathbf{x}$ through the composed mapping $d_{L}^{h}=\left(\mathbf{d}_{L} \circ \mathbf{v}_{L}\right)(\mathbf{x})$. This means that given an amount of production $\mathbf{x}$ we obtain a certain amount of home value added $\mathbf{v}_{L}^{h}$ which, in turn, determines the home final demand of the economy $\mathbf{d}_{L}^{h}$. Similarly, in the case of the extended Ghosh model (with variables now identified by the submarker $G$ ), we have that value-added is a function of output $\mathbf{x}$ through the composed mapping $v_{G}^{h}=\left(\mathbf{v}_{G} \circ \mathbf{d}_{G}\right)(\mathbf{x})$. Notice that from output $\mathbf{x}$ we obtain now internal demand $\mathbf{d}_{G}^{h}$ which yields the home value-added of the economy $\mathbf{v}_{G}^{h}$. In particular, the way that this mapping is specified in Guerra and Sancho (2011) in their extended Ghosh model, for $j=1,2$, can be seen to be the following:

$$
\begin{aligned}
& d_{G}^{h}(j)=d_{G}\left(x_{G}(j)\right)=\alpha(j) \cdot x_{G}(j) \\
& v_{G}^{h}(j)=v_{G}\left(d_{G}^{h}(j)\right)=\beta(j) \cdot\left(d_{G}^{h}(1)+d_{G}^{h}(2)\right)
\end{aligned}
$$

The first mapping tell us that home final demand in each sector is a fixed share, $\alpha(j)$, of total sectorial production, while the second mapping stipulates that value-added in each sector is a fixed share, $\beta(j)$, of the total amount of home final demand. Putting the two mappings together we obtain:

$$
v_{G}^{h}(j)=v_{G}\left(d_{G}\left(x_{G}(j)\right)=\beta(j) \cdot\left(\alpha(1) \cdot x_{G}(1)+\alpha(2) \cdot x_{G}(2)\right)\right.
$$

Notice that the value-added in sector $j$ depends on a fraction, $\beta(j)$, of the weighted sum of the total production in each sector. The sum of those fractions must be equal to one.

The specific mapping for the extended Leontief model is logically defined, for $j=1,2$, as the symmetric counterpart of the extended Ghosh equations, where $\alpha^{\prime}(j)$ and $\beta^{\prime}(j)$ are the corresponding shares:

$$
\begin{aligned}
& v_{L}^{h}(j)=\mathbf{v}_{L}\left(x_{L}(j)\right)=\alpha^{\prime}(j) \cdot x_{L}(j) \\
& d_{L}^{h}(j)=d_{L}\left(v_{L}^{h}(j)\right)=\beta^{\prime}(j) \cdot\left(v_{L}^{h}(1)+v_{L}^{h}(2)\right)
\end{aligned}
$$

Substituting the first expression into the second one we obtain:

$$
d_{L}^{h}(j)=d_{L}\left(\mathbf{v}_{L}\left(x_{L}(j)\right)=\beta^{\prime}(j) \cdot\left(\alpha^{\prime}(1) \cdot x_{L}(1)+\alpha^{\prime}(2) \cdot x_{L}(2)\right)\right.
$$

Comparing equations (2) and (3a) we observe that in the extended L model the only variable affecting, in a positive relation, value-added in sector $j$ is production in sector $j$. In the extended $\mathrm{G}$ model, as we said before, the value-added of a sector is affected by the production of the two sectors. This is a key difference between the two extended models concerning the relationship between production and value-added. The other key difference 
between the two models concerns the relationship between the production of a sector and its domestic demand. In the extended L model, we see in expression (4) that domestic demand for sector $j$ depends on the production levels of the two sectors, while in the extended G model, equation (1a) shows that it depends exclusively on the production of sector $j$. The two different ways of endogenizing the relevant variables discussed above gives rise to the different anomalies in the equilibrium behavior of the two models.

This is not, however, the end of this story. When we compare the behavior of the two models in the assignment of final domestic demand, we can see in the extended L model the symmetrical counterpart of what we have being saying about the relationship between sectorial value-added and total production in the extended G model.

In the extended $\mathrm{G}$ model we find that there will always be a positive relationship between increasing production, in a given sector, and increasing domestic final demand for the good of that sector. However, in the extended L model we may have an increase in production and value-added in a given sector with a lower domestic demand for the good of that sector. This biased property of the extended L model will happen as often as the biased property of the extended $\mathrm{G}$ model concerning value-added and production at the sectorial level.

It is nonetheless quite surprising that an increase in output and value-added in a given sector will not translate into additional domestic demand for the same good. In general, and under an income normality assumption, more available income should generate more demand for all goods, rather than less for some. Even the recourse to argue some consumption substitution will not do since in the standard linear models this is axiomatically ruled out.

Finally, we also want to comment on the unwanted possibility, shared by the two models, of having an increase in total aggregate production while total value-added decreases in the economy, or viceversa. By looking at equation (2), we can see that if the $\alpha$ coefficients in this equation are equal, then we have a positive relationship between aggregate output and aggregate value-added. Otherwise, as can be seen Oosterhaven (2012) numerical example, the unwanted property becomes indeed real. In fact, we will see from our $2 \times 2$ analysis that we also are able to construct examples with the undesirable property for the extended $G$ model. Of course, the same happens for the extended L model.

By looking at expression (3a) for the extended L model, we can also conclude that if the $\alpha^{\prime}$ coefficients have the same value, aggregate production is positively related to aggregate value-added, and so the unwanted property disappears. Outside this stringent equality condition, we can also show the presence of the corresponding anomaly in the extended L model.

\subsection{Solving the extended models for the $2 \times 2$ case}


We start substituting expressions (2) and (4) into (G.3) and (L.3), respectively. This substitutions yield extended coefficient matrices $\hat{\mathbf{A}}$ and $\hat{\mathbf{B}}$, which can then be used to solve for the values of output $\mathbf{x}$ in both models. We obtain the following expressions, where we use again the markers $L$ and $G$ to distinguish the solutions:

$$
\begin{aligned}
& \mathbf{x}_{\mathrm{L}}=(\mathbf{I}-\hat{\mathbf{A}})^{-1} \cdot \mathbf{d}^{\mathbf{f}} \\
& \mathbf{x}_{\mathrm{G}}=(\mathbf{I}-\hat{\mathbf{B}})^{-1} \cdot \mathbf{v}^{\mathbf{f}}
\end{aligned}
$$

The inverse matrices $(\mathbf{I}-\hat{\mathbf{A}})^{-1}=[\hat{a}(i, j)]$ and $(\mathbf{I}-\hat{\mathbf{B}})^{-1}=[\hat{b}(i, j)]$ can be seen to have the following elements:

$$
\begin{aligned}
& \hat{a}(1,1)=1-\left(a(2,2)+\beta^{\prime}(2) \cdot \alpha^{\prime}(2)\right) / \Delta_{L} \\
& \hat{a}(1,2)=\left(a(1,2)+\beta^{\prime}(1) \cdot \alpha^{\prime}(2)\right) / \Delta_{L} \\
& \hat{a}(2,1)=\left(a(2,1)+\beta^{\prime}(2) \cdot \alpha^{\prime}(1)\right) / \Delta_{L} \\
& \hat{a}(2,2)=1-\left(a(1,1)+\beta^{\prime}(1) \cdot \alpha^{\prime}(1)\right) / \Delta_{L} \\
& \hat{b}(1,1)=1-(b(2,2)+\beta(2) \cdot \alpha(2)) / \Delta_{G} \\
& \hat{b}(1,2)=(b(2,1)+\beta(2) \cdot \alpha(2)) / \Delta_{G} \\
& \hat{b}(2,1)=(b(1,2)+\beta(2) \cdot \alpha(1)) / \Delta_{G} \\
& \hat{b}(2,2)=1-(b(1,1)+\beta(1) \cdot \alpha(1)) / \Delta_{G},
\end{aligned}
$$

where $\Delta_{L}$ and $\Delta_{G}$ are the determinants of matrices $(\mathbf{I}-\hat{\mathbf{A}})$ and $(\mathbf{I}-\hat{\mathbf{B}})$, respectively. Since the economies have to be productive, we may assume, invoking the Hawking-Simon conditions, that both determinants are positive.

We will assume now, as a way of normalizing the total value of the exogenous variables from both models that the total amount of foreign demand and foreign inputs are unitary:

$$
d^{f}(1)+d^{f}(2)=v^{f}(1)+v^{f}(2)=1
$$

This is the same assumption as in Oosterhaven (2012) when he redistributes resources among sectors.

\subsubsection{Sectorial analysis of equilibrium}


It is now a matter of performing some algebra to verify that the equilibrium solution for production and value-added in the extended $\mathrm{G}$ model is as follows:

$$
\begin{aligned}
& x_{G}^{*}(1)=\hat{b}(1) \cdot v^{f}(1)+\hat{b}(1,2) \\
& x_{G}^{*}(2)=\hat{b}(2) \cdot v^{f}(1)+\hat{b}(2,2) \\
& v_{G}^{h} *(1)=\beta(1) \cdot \Omega(0) \cdot v^{f}(1)+\Omega(1) \\
& v_{G}^{h} *(2)=\beta(2) \cdot \Omega(0) \cdot v^{f}(1)+\Omega(1)
\end{aligned}
$$

where it can be seen ${ }^{2}$ that:

$$
\begin{aligned}
& \hat{b}(1)=(1-\bar{b}(2)-\alpha(2)) / \Delta_{G} \\
& \hat{b}(2)=-(1-\bar{b}(1)-\alpha(1)) / \Delta_{G} \\
& \Omega(0)=(\alpha(2) \cdot \bar{b}(1)-\alpha(1) \cdot \bar{b}(2)+\alpha(1)-\alpha(2)) / \Delta_{G}
\end{aligned}
$$

and $\bar{b}(1)$ and $\bar{b}(2)$ are the sum of columns 1 and 2, respectively, in matrix B. A quick substitution also shows:

$$
\Omega(0)=\alpha(1) \cdot \hat{b}(1)+\alpha(2) \cdot \hat{b}(2)
$$

Similar algebra manipulations and notational conventions to the above ones would yield equilibrium output in the extended L model as:

$$
\begin{aligned}
& x_{L}^{*}(1)=\hat{a}(1) \cdot d^{f}(1)+\hat{a}(1,2) \\
& x_{L}^{*}(2)=\hat{a}(2) \cdot d^{f}(1)+\hat{a}(2,2)
\end{aligned}
$$

with home final demand being:

$$
\begin{aligned}
& d_{L}^{h} *(1)=\beta^{\prime}(1) \cdot\left(\Omega^{\prime}(0) \cdot d^{f}(1)+\Omega^{\prime}(1)\right) \\
& d_{L}^{h *(2)}=\beta^{\prime}(2) \cdot\left(\Omega^{\prime}(0) \cdot d^{f}(1)+\Omega^{\prime}(1)\right)
\end{aligned}
$$

\footnotetext{
${ }^{2}$ The specifics of $\Omega(1)$ in (7) and (8) are omitted since this magnitude plays no role in the subsequent analysis. The same applies later to $\Omega^{\prime}(1)$.
} 
In these expressions we would have:

$$
\begin{aligned}
& \hat{a}(1)=\left(1-\bar{a}(2)-\alpha^{\prime}(2)\right) / \Delta_{L} \\
& \hat{a}(2)=-\left(1-\bar{a}(1)-\alpha^{\prime}(1)\right) / \Delta_{L} \\
& \Omega^{\prime}(0)=\left(\alpha^{\prime}(2) \cdot \bar{a}(1)-\alpha^{\prime}(1) \cdot \bar{a}(2)+\alpha^{\prime}(1)-\alpha^{\prime}(2)\right) / \Delta_{L}= \\
& =\alpha^{\prime}(1) \cdot \hat{a}(1)+\alpha^{\prime}(2) \cdot \hat{a}(2)
\end{aligned}
$$

We are now in a position to compare the equilibrium relationships for the behavior of sectorial production and sectorial value-added in the extended $G$ model, and sectorial production and sectorial home final demand in the extended L model. We will see that these relationships run in parallel (or symmetric) fashion in both models.

In the extended $\mathrm{G}$ model we first check expressions (5) to (8). Notice that the exogenous variable affecting the two endogenous variables in sectors $j=1,2$, output $x(j)$ and home value-added $v^{h}(j)$ is external value-added in sector $1, v^{f}(1)$. Therefore any change in $v(1, f)$ will affect the endogenous variables this way:

$$
\begin{aligned}
& \frac{\partial x_{G}(1)}{\partial v^{f}(1)}=\hat{b}(1) \\
& \frac{\partial x_{G}(2)}{\partial v^{f}(1)}=\hat{b}(2) \\
& \frac{\partial v_{G}^{h}(1)}{\partial v^{f}(1)}=\beta(1) \cdot \Omega(0) \\
& \frac{\partial v_{G}^{2}(2)}{\partial v^{f}(1)}=\beta(2) \cdot \Omega(0)
\end{aligned}
$$

and the signs of these derivatives become the key factor in the discussion. It can be readily proved that it is always the case that $\hat{b}(1)>0$ and $\hat{b}(2)<0$. From expression (9), since $\Delta_{G}>0$ the sign of $\hat{b}(1)$ will be determined simply by the sign of $1-\bar{b}(2)-\alpha(2)$. Take the second equation of (L.1) and divide it by $x(2)$ to obtain $b(2,1)+b(2,2)+\alpha(2)<1$, provided external final demand is positive; from here we immediately find that $\Delta_{G} \cdot \hat{b}(1)=1-b(2,1)-b(2,2)-\alpha(2)=1-\bar{b}(2)-\alpha(2)>0$. A similar argument would show that $\hat{b}(2)<0$.

Endogenous home value-added, however, will always move in the same direction in both sectors. This is so because $\beta(j)>0$ and thus it all rests on the sign of parameter $\Omega(0)$, which can be seen to have any sign, either positive or negative. If $\Omega(0)>0$, home valueadded will surely rise in both sectors at once. If $\Omega(0)<0$ there would apparently be an expost input substitution between home and foreign inputs. This seems unlikely to be the 
case in practical terms since no substitution is allowed in the linear models. In any case we will always have one sector's value-added going in the opposite direction of its production. However, by the nature of the endogenizing rules, the direction of change in sectorial output, will always agree with the direction of change in sectorial home final demand.

This alleged inconsistent behavior of the extended $\mathrm{G}$ model is not possible in the extended $\mathrm{L}$ model and the reason is equation (3a). However, and for exactly the same formal reasons, we may find in equilibrium a likewise anomalous relationship in the extended $\mathrm{L}$ model. In this case the anomaly is between the direction of change in sectorial output (or value-added) and home final demand for that sector.

That this is indeed the case can be ascertained if we look at the equations (12) though (15) and their corresponding derivatives:

$$
\begin{aligned}
& \frac{\partial x_{L}(1)}{\partial d^{f}(1)}=\hat{a}(1) \\
& \frac{\partial x_{L}(2)}{\partial d^{f}(1)}=\hat{a}(2) \\
& \frac{\partial d_{L}^{h}(1)}{\partial d^{f}(1)}=\beta^{\prime}(1) \cdot \Omega^{\prime}(0) \\
& \frac{\partial d_{L}^{h}(2)}{\partial d^{f}(1)}=\beta^{\prime}(2) \cdot \Omega^{\prime}(0)
\end{aligned}
$$

The same line of reasoning that we took in the extended $\mathrm{G}$ model can also be made now for the extended L model. The signs of the parameter affecting equilibrium production in each sector will be once again different and it can be seen that it is always the case that $\hat{a}(1) \geq 0$ and $\hat{a}(2) \leq 0$. Since the proof is formally similar to the one given above for the extended $\mathrm{G}$ model, it is omitted here. We see from (14) and (15), however, that there is only one parameter affecting the sign of the derivatives of internal demand in both sectors, i.e. $\Omega^{\prime}(0)$. Regardless of the sign of $\Omega^{\prime}(0)$, it must be different from the sign of one of the two previous parameters affecting sectorial production (and value-added). Hence, we will observe in the extended L model increases in sectorial production (and value-added) along with a decrease in the internal (home) demand of that same sector. Notice the anomaly: an increase in final foreign demand for a given good may induce a reduction in the home demand for both goods, even if production goes up for some good.

\subsubsection{Aggregate analysis of the equilibrium}

We now consider the equilibrium relationships between aggregate output and aggregate exogenous changes.

Aggregate equilibrium output and value-added, in each of the G and L models, can be seen to be as follows: 


$$
\begin{aligned}
& x_{G}^{*}=\hat{b} \cdot v^{f}(1)+\hat{b}(1,2)+\hat{b}(2,2) \\
& v_{G}^{*}=\Omega(0) \cdot v^{f}(1)+\Omega(1) \\
& x_{L}^{*}=\hat{a} \cdot d^{f}(1)+\hat{a}(1,2)+\hat{a}(2,2) \\
& v_{L}^{*}=\Omega^{\prime}(0) \cdot d^{f}(1)+\Omega^{\prime}(1)
\end{aligned}
$$

where we would now have:

$$
\begin{aligned}
& \hat{b}=(\bar{b}(1)-\bar{b}(2)+\alpha(1)-\alpha(2)) / \Delta_{G}=\hat{b}(1)+\hat{b}(2) \\
& \hat{a}=\left(\bar{a}(1)-\bar{a}(2)+\alpha^{\prime}(1)-\alpha^{\prime}(2)\right) / \Delta_{L}=\hat{a}(1)+\hat{a}(2)
\end{aligned}
$$

Again, for our purposes here, we do not need to specify the expressions for $\Omega(1)$ and $\Omega^{\prime}(1)$. For the extended G model, as we can see from (19) and (20), a change in the same direction in total aggregate production and total value-added will require that the parameters affecting $v^{f}(1)$ in (19) (i.e. $\hat{b}$ ) and in (20) (i.e. $\Omega(0)$ ) both have the same sign. We now show that this is not going to be the case always and why these parameters may have different signs.

Let us begin by considering the product of the two relevant parameters:

$$
P=\hat{b} \cdot \Omega(0)=(\bar{b}(1)-\bar{b}(2)+\alpha(1)-\alpha(2)) \cdot(\alpha(2) \cdot \bar{b}(1)-\alpha(1) \cdot \bar{b}(2)+\alpha(1)-\alpha(2)) / \Delta_{G}^{2}
$$

If the sign of this product $\mathrm{P}$ is positive, then we will have the same direction of change in the aggregate variables. It is easy to see that the following statements are true:
a) If $\alpha(1)=\alpha(2)$ or $\bar{b}(1)=\bar{b}(2)$ then $P>0$.
b) If $\alpha(1)=\alpha(2)$ and $\bar{b}(1)=\bar{b}(2)$ then $\hat{b}=0$ and $\Omega(0)=0$. Hence $P=0$.
c) If $\alpha(2)=\bar{b}(1)$ and $\alpha(1)=\bar{b}(2)$ then $\hat{b}=0$ and if $\alpha(1)>(<) \alpha(2)$ then $\Omega(0)>(<) 0$. Either way $P=0$.

We have to consider now the case $\hat{b}>0$ and $\Omega(0)<0$. Notice now:

d) $\hat{b}>0$ if and only if $\bar{b}(2)+\alpha(2)<\bar{b}(1)+\alpha(1)$

e) $\Omega(0)<0$ if and only if $\alpha(2) \cdot \bar{b}(1)+\alpha(1)<\alpha(1) \cdot \bar{b}(2)+\alpha(2)$

These two previous inequalities will be satisfied simultaneously whenever the following ones hold: 


$$
\alpha(2) \cdot \bar{b}(1)+\alpha(1)<\alpha(1) \cdot \bar{b}(2)+\alpha(2)<\bar{b}(2)+\alpha(2)<\bar{b}(1)+\alpha(1)
$$

The following numerical example satisfies these inequalities:

$$
\alpha(1)=0.15 \quad \alpha(2)=0.20 \quad \bar{b}(1)=0.25 \quad \bar{b}(2)=0.13
$$

From these figures, it is easily checked that:

$$
\begin{aligned}
& \hat{b}(1)=2.713>0 \\
& \hat{b}(2)=-2.429<0 \\
& \hat{b}=\hat{b}(1)+\hat{b}(2)=0.283>0 \\
& \Omega(0)=\alpha(1) \cdot \hat{b}(1)+\alpha(2) \cdot \hat{b}(2)=-0.079<0 \\
& \Delta_{G}=0.247>0
\end{aligned}
$$

with values for the Ghosh coefficient matrix $\mathbf{B}$ as follows:

$$
\mathbf{B}=\left[\begin{array}{ll}
0.15 & 0.05 \\
0.10 & 0.08
\end{array}\right]
$$

According to our analysis, and for the previous parameter values, an increase in $v(1, f)$ will increase production in sector one, decrease production in sector 2 , and decrease valueadded in both sectors. Total production will increase, but total value-added will decrease, and thus the anomalies in the extended $\mathrm{G}$ model are present. The same type of example can be found for the extended Leontief model: in this model, where unwanted property shows up when changes in total production (and total value-added) go in the opposite direction of changes in total domestic demand.

\section{Alternative ways to make value-added and final demand endogenous}

We have discussed in the previous section some anomalous implications that may follow from the standard extension implementation of the Leontief and Ghosh models. As we have seen, both these models may have unwanted properties in the behavior of fundamental equilibrium variables, both at the sectorial and the aggregate level. Here we wish to consider some other specific function that relates those variables in a sensible economic way and, at the same time, avoids the inconsistencies found under the previous rules.

We may have the following proposal where for each sector its endogenous variables are related only to the other variable of the same sector. 
New extended Ghosh model "rules":

$d^{h}(j)=\alpha(j) \cdot x(j), \quad j=1,2 ; \quad v^{h}(j)=d^{h}(j), j=1,2$.

New extended Leontief model "rules":

$v^{h}(j)=\alpha(j) \cdot x(j), \quad j=1,2 ; \quad d^{h}(j)=v^{h}(j), \quad j=1,2$.

Under these two rules, both models will behave in a "proper" way. We will have by construction that in each sector value-added, final home demand and total production will all change in the same direction in response to an external shock. At the aggregate level and under the present rules, however, we may still have inconsistent behavior between the direction of the change in total production and total value-added (or total internal demand).

In effect, going through the same procedure as in section 2, we may solve for the equilibrium solution of the endogenous variables as a function of the exogenous ones. In particular, for the Ghosh model, after the usual algebra we find the following fundamental derivatives at the sectorial equilibrium values:

$$
\begin{aligned}
& \frac{\partial x_{G}(1)}{\partial v^{f}(1)}=\hat{b}(1)>0 \\
& \frac{\partial x_{G}(2)}{\partial v^{f}(1)}=\hat{b}(2)<0 \\
& \frac{\partial v_{G}^{h}(1)}{\partial v^{f}(1)}=\alpha(1) \cdot \hat{b}(1)>0 \\
& \frac{\partial v_{G}^{h}(2)}{\partial v^{f}(1)}=\alpha(2) \cdot \hat{b}(2)<0
\end{aligned}
$$

while at the aggregate level we find the following expressions:

$$
\begin{aligned}
& \frac{\partial x_{G}}{\partial v^{f}(1)}=\hat{b}=\hat{b}(1)+\hat{b}(2) \\
& \frac{\partial v_{G}}{\partial v^{f}(1)}=\Omega(0)=\alpha(1) \cdot \hat{b}(1)+\alpha(2) \cdot \hat{b}(2)
\end{aligned}
$$

As can be seen, at the sectorial level, all derivatives have the correct sign. However, the sign of the derivatives, at the aggregate level, are ambiguous. Hence, we still have the same problem that we had under the previous rules, and so the same analysis made in section 3.4 applies here. In that analysis, we showed that one of the sufficient conditions to avoid the unwanted aggregate property, in both models, is to assume that the $\alpha$ 's are the same for all sectors. A parallel analysis, to the previous one, can be made for the new extended Leontief model, which would lead to the same type of equations and conclusions. 
In both models, the new defined rules tell us that, the facto, the value-added of a sector is a positive constant fraction of the total production of that sector. Since the input-output coefficients are also a constant fraction of production, our rules have a sound economic property resembling the assumptions made empirically to define the production function of a sector. Now, both models share this property. We also see that value-added, in a given sector, is equal in both models to final demand. We interpret this assumption as a way to as measure the value-added of a sector in terms of the product produced by such sector. We see this very often in neoclassical economics when we measure the value of wages that a firm is paying to its labor input in terms of its final product. In the neoclassical analysis, the marginal product of the input determines its unit price (in production units). Here, in our framework, we just take a fraction of the production.

\section{Conclusions}

By using a $2 \times 2$ commodity framework, we have formally explored the roots of the alleged anomalies in the extended Ghosh model first pointed out by Oosterhaven (2012) by means of some numerical examples. We find that systematically we may have a positive change in the production of one sector with a decrease in its value added. We have proved that some other anomalies also lurk in the extended Leontief model. Namely, we can have some sector where, systematically, a positive change in output goes along with a negative change in its final domestic consumption. Furthermore, both models, share the unwanted property that it is possible to have a negative relationship between aggregate total production and aggregate total value added. The odd behavior of these two classical extended models is hard to find in reality. The paper is a methodological one, thus the finding regarding the biased behavior is independent of the data base underlying the models - be it a text book example or a real world, empirical data base. The systematic biases will always take place within these models' bounds. The implication for empirical linear analysis seems to point out to a misspecification of the general equilibrium feedbacks implicit in the standard endogeneization of the extended linear models.

This need not be the case, however, if other assumptions are considered. We have introduced, in section 4, a new set of rules to make endogenous the relation between value added and final demand. Under these new rules, at the sectorial level we are able to overcome the previous criticisms for both models, but not at the aggregate level. Nevertheless, some simple hypothesis can also overcome the anomalous aggregate behavior of the two models both under the new as well as the old rules.

\section{References}

De Mesnard, L. (2009), Is the Ghosh model interesting?, Journal of Regional Science, 49, pp. 361-372. 
Dietzenbacher, E. (1997), In vindication of the Ghosh Model: A reinterpretation as a price model, Journal of Regional Science, 37(4), pp. 629-651.

Ghosh, A. (1958), Input-output approach in an allocation system. Economica, 25, pp. 5864.

Guerra, A.I. and F. Sancho (2011), Revisiting the original Ghosh model: can it be made more plausible?. Economic Systems Research, vol. 23(3), pp. 319-328.

Guerra, A.I. and F. Sancho (2012), A non-possibility theorem for joint stability in interindustry models. Applied Mathematics and Computation, 219(8), pp. 4045-4048

Leontief, W. (1936), Quantitative input and output relations in the economic system of the United States. Review of Economics and Statistics, 28, pp. 105-25.

Miller, R.E and P.D. Blair (2009), Input-output Analysis: Foundations and Extensions, Cambridge University Press, New York.

Oosterhaven, J. (2012), Adding Supply-Driven Consumption Makes the Ghosh Model Even More Implausible, Economic Systems Research, vol. 24(1), pp. 101-111.

Oosterhaven, J. (1988), On the Plausibility of the Supply-driven Input-output Model, Journal of Regional Science, 28, pp. 203-217.

Oosterhaven, J. (1989), The Supply-driven Input-output Model: A New interpretation but still Implausible, Journal of Regional Science, 29, pp. 459-465. 